2014

\title{
Brain Control of Functional Reach in Healthy Adults and Stroke Survivors
}

Janis J. Daly

Neurology and Research Service

Ken Hrovat

Brain Rehabilitation Research Center

John Holcomb

Cleveland State University, j.p.holcomb@csuohio.edu

Svetlana Pundik

Case Western Reserve University

Follow this and additional works at: https://engagedscholarship.csuohio.edu/scimath_facpub

Part of the Mathematics Commons

How does access to this work benefit you? Let us know!

\section{Repository Citation}

Daly, Janis J.; Hrovat, Ken; Holcomb, John; and Pundik, Svetlana, "Brain Control of Functional Reach in Healthy Adults and Stroke Survivors" (2014). Mathematics Faculty Publications. 167.

https://engagedscholarship.csuohio.edu/scimath_facpub/167

This Article is brought to you for free and open access by the Mathematics and Statistics Department at EngagedScholarship@CSU. It has been accepted for inclusion in Mathematics Faculty Publications by an authorized administrator of EngagedScholarship@CSU. For more information, please contact library.es@csuohio.edu. 


\title{
Brain control of functional reach in healthy adults and stroke survivors
}

Janis J. Daly, Ken Hrovat, John Holcomb and Svetlana Pundik

\begin{abstract}
.
Purpose: Recovery of the most basic shoulder-flexion/elbow-extension components of functional reach is critical for effective arm function following stroke. In order to understand the mechanisms of motor recovery, it is important to characterize the pattern of brain activation during the reach task.

Methods: We evaluated 11 controls and 23 moderately to severely impaired chronic stroke survivors ( $>6$ months), with impaired shoulder flexion and elbow extension. Measures were acquired for Arm Motor Ability Test (AMAT) and functional Magnetic Resonance Imaging (fMRI) during the basic shoulder/elbow reach.

Results: First, in controls, lateralization of fMRI signal during the reach task was less pronounced in comparison to other tasks, and even further diminished after stroke $(p<0.05)$. Second, for the stroke group, centroid locations, for specific ipsilesional (contralateral to working limb) motor-sensory regions and for contralesional (ipsilateral to working arm) somatosensory and SMA regions, were significantly more distant from the centroid location of average healthy controls $(p<0.05)$. Third, both greater activation volume and greater degree of signal intensity were correlated with better motor function in stroke survivors. Conclusions: These findings can be useful in guiding the development of more targeted brain training methods for recovery of impaired reach coordination.
\end{abstract}

Keywords: Stroke, arm function, motor control, brain function, functional MRI, brain activation

\section{Introduction}

One of the most important basic functions of the upper extremity is functional reach, that is, translation of the upper limb through space to the desired location so that the hand can perform functional tasks. From the considerable research efforts regarding studies of healthy control subjects, we are able to appreciate the complexity of the central neural pathways involved in the control of shoulder/elbow reach and hand grasp movements (Himmelbach et al., 2013; Konen et al., 2013; Bernier et al., 2012; Gallivan et al., 2009; Cavina-Pratesi et al., 2010; Desmurget et al., 2001; Kertzman et al., 1997; Gallivan et al., 2013; Dean et al., 2012).

After stroke, arm and hand coordination can be mildly to severely impaired, with resulting disability and high cost to individuals and society (Carod-Artal and Egido 2009; Harvey et al., 1998). For stroke survivors, almost all of the upper limb studies were limited to distal limb tasks, such as finger flexion/extension (Askim et al., 2009; Calautti et al., 2010;Carey et al., 
2005; Jang et al., 2004; Loubinoux et al., 2007; Marshall et al., 2000; Small et al., 2002; Zemke et al., 2003) or grasp (Fujii and Nakada 2003; Kokotilo et al., 2010; Ward et al., 2003). These studies of hand function are important; at the same time, the hand is not useful unless it can be translated through the workspace to the desired location for execution of the hand tasks.

There have been only a few studies of fMRI-derived measures of brain control of shoulder movements in stroke survivors. One was a two-case study of a reach movement, with functional connectivity measured before and after a treatment for these two subjects (Sergi et al., 2011). A second study compared shoulder adduction vs elbow flexion cortical representation using electroencephalographic signal (EEG) (Yao et al., 2009); EEG provides good precision in terms of brain latencies, but does not have good resolution with regard to location of brain activations. Finally, two other studies focused on the relatively simple movements of shoulder abduction (Feydy 2002; a two-case report) or internal rotation of the humerus (Cramer and Crafton 2006), leaving uninvestigated, the very basic reach components of shoulder/flexion and elbow extension in stroke survivors, with regard to fMRI measures of the location of brain function.

It is important to gain an understanding of the brain control of the functional reach task and the impairment in brain control of this task after stroke. Shoulder flexion and elbow extension are among the most basic components of functional reach. Given the paucity of information on brain control of these basic reach components (referred to as 'functional reach', for simplicity, throughout the remainder of the manuscript), the two-fold purpose of this study was to: 1) characterize the pattern of brain activation during execution of functional reach in healthy adults and chronic, moderately to severely impaired stroke survivors in the chronic phase ( $>6$ months); and 2 ) identify brain activation correlates with upper limb functional performance in stroke survivors.

\section{Methods}

\subsection{Subjects}

We enrolled 23 individuals with chronic stroke (stroke onset $>6$ months) and 11 healthy adults. The inclusion criteria for stroke subjects were as follows: age $>21$ yrs, single stroke, at least a trace grade in man- ual muscle testing for movement in the stroke-affected arm, and no contra-indications for MRI. Subjects were assessed for impairment level according to the FuglMeyer test (Duncan et al., 1983; Fugl-Meyer et al., 1975). The study protocol was approved by the Institutional Review Board in the Medical Center; informed written consent was obtained for each subject.

\subsection{MRI data acquisition}

MRI was acquired using a Siemens Symphony 1.5 $\mathrm{T}$ system with a circularly polarized head coil and an interleaved multi-slice gradient-echo echoplanar imaging (EPI) sequence. Both T1-weighted anatomical and blood-oxygenation level dependent (BOLD) images were collected in the axial plane. BOLD data acquisition parameters were as follows: in-plane resolution was $3 \times 3 \mathrm{~mm}$, repetition time (TR) was $3.87 \mathrm{~s}$, echo time (TE) was $50 \mathrm{~ms}$, flip angle was 90 degrees, and the number of axial slices through the entire cerebrum was 36 . For $\mathrm{T} 1$ images, in-plane resolution was $1 \times 1 \mathrm{~mm}, \mathrm{TR}=2.16 \mathrm{~s}, \mathrm{TE}=3.45 \mathrm{~ms}$, and flip angle was 15 degrees.

BOLD data was acquired in a block design with alternating move and rest periods (10scans/period, 40 seconds/block); rest/move cycles were repeated 5 times; each move block consisted of 5 move cycles; the paradigm started and ended with a rest block. For every block and movement trial, the resting position was with humerus resting flat on the MRI bed and forearm/hand cradled and supported in the movement guide. The reach task for the involved arm was performed by flexing the shoulder/extending the elbow, sliding the arm along a custom-designed wooden movement guide placed at a 30 degree angle with respect to the horizontal bed (Fig. 1); the forearm was placed in a pronated position, and the hand was placed, with the mid-palm leaning on the dowel-stick palm support, with fingers in the relaxed, neutral position, resting on the movement guide; the weight of the hand and forearm were sufficient to maintain the arm and hand in this position, so that grasp was not demanded for this shoulder flexion/elbow extension task. For a few subjects, the forearm and hand did not naturally remain in the supported, relaxed position, during the motor task; in that case, the hand was secured in the desired, relaxed position, to the palm-rest using a soft strap. This position was maintained for all subjects during rest and movement. Friction in the system was minimized by using soft polished wooden materials. Commands, at 


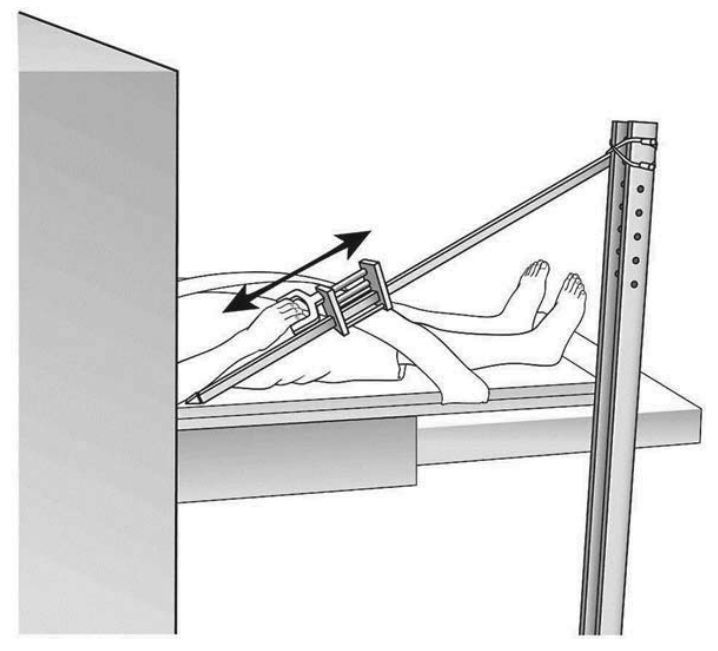

Fig. 1. fMRI Setup for shoulder/elbow motor task.

a timed frequency, were used to cue the subject to begin the motor task and to rest. Practice sessions were performed the day before (outside of the MRI department) and on the day of testing (in the MRI room) in order to ensure that the motor tasks were understood and accurately performed. Using methods described elsewhere (Daly et al., 2008), the potential for confounding mirror movements in the uninvolved, non-tested arm was monitored through the use of an MRI-compatible EMG data acquisition system (BrainVision LLC, Morrisville, NC, USA), with monitoring of the following uninvolved limb muscles: anterior deltoid, triceps, biceps, wrist and finger flexors and extensors. Scans were discarded in the event that they were associated with undesired activation of the uninvolved, non-tested limb.

During data acquisition, we found that head movement was limited to $<0.4 \mathrm{~mm}$ of translation in the $\mathrm{x}, \mathrm{y}$, and $\mathrm{z}$ directions and $<0.4^{\circ}$ of rotation; to accomplish this, we used the following methods: 1) Dense sponge materials were placed within the head coil for stabilization of the head; position and amount of the sponge materials was customized according to given head size; 2) A strap system was constructed to stabilize the torso. To control for rotational or other movements of the upper torso, a strap was secured to the plinth proximal to the shoulder and extended across the upper torso clavicular region, subsequently running diagonally, to the opposite side of the torso and secured to the plinth just distal to the waist; this strap system was applied to both sides of the body. This strap secured the torso, but allowed free movement of the humerus with respect to the glenoid fossa for the shoulder movements of flexion and extension; 3) The motor task was practiced prior to scanning, using EMG signal to monitor undesired muscle activation in the non-tested upper limb and to instruct the participant as to the level of effort needed in order to execute the desired task.

\subsection{MRI data analysis}

The approach to MRI analysis was consistent with other successful studies of stroke motor behavior (Cramer and Crafton 2006; Dong et al., 2007; von et al. 2009). MRI data were processed and analyzed using the Statistical Parametric Mapping (SPM5) package (Wellcome Department of Imaging Neuroscience at University College London, UK), along with custom in-house software analysis packages designed by our lab using the MATLAB (The MathWorks, Inc., Natick, MA) technical computing environment.

The fMRI data analysis included several temporal and spatial pre-processing steps. The initial 2 scans were discarded for each of the rest and move blocks to account for the delay in the hemodynamic response. Slice-timing and head motion corrections were performed. Anatomical and BOLD images were co-registered. Extraneous non-brain signal was excluded using brain parenchyma segmentation. Data were normalized to a standard template based on the Montreal Neurological Institute (MNI) reference data. Spatial smoothing was performed with a $6 \mathrm{~mm}^{3}$, full-width at half maximum Gaussian kernel. For the analyses, images were right/left flipped in order to align the lesion hemispheres which were all contralateral to the tested arm (Nair et al., 2007; Loubinoux et al., 2007; Crafton et al., 2003). All control subjects performed the task with the dominant arm. In $70 \%$ of the stroke group, the dominant side was affected by stroke. The shoulder/elbow reach task is a gross proximal arm movement, and at the relatively low effort level for this task, would not be influenced by arm dominance (Macedo and Magee 2008; Acuna et al., 2010).

We studied brain activation measures within 5 functional sensorimotor regions of interest (ROI), as follows: (1) primary motor region (Brodmann area (BA) 4); (2) somatosensory region (BA 1, 2, 3); (3) lateral premotor area (LPM) (lateral surface of BA 6) (Picard and Strick 2001); (4) Supplementary Motor Area (SMA) proper (medial portion BA 6 that is poste- 
rior to the anterior commissure line) (Picard and Strick 1996); and (5) Posterior parietal (PP) region (BA 5, 7). Contralateral and ipsilateral hemispheres were considered separately.

ROI selection was based on the role in motor function. We used brain atlas templates to identify the ROIs; the images were transformed into a standard MNI template. If lesion intruded into ROIs, we inspected the MNI transformation to ensure no distortion of ROI. Then, pre-existing anatomical masks provided by SPM software were applied to select voxels corresponding to the pre-specified ROIs. We carefully inspected fMRI activation maps in relation to the lesions to determine whether there were voxel activations in the cavity of the lesion; for 21 of the 23 subjects, we found no voxel activity in the lesion area. For two subjects, there was activity identified around the periphery of the lesion. From our inspection of the data, it appeared that this activation was in neural tissue, and we treated it as such; this was minimal in volume and intensity, in comparison to other activation for those two subjects. The same individual processed the data for all subjects.

Each of the 5 ROIs was utilized in comparing stroke versus healthy adult, according to brain activation patterns during the functional reach task. In addition, brain activation measures within each of the 5 regions of interest were utilized in investigation of brain activation pattern correlates with a measure of complex functional task performance.

\subsection{Functional motor measure}

We used the Arm Motor Ability Test (AMAT) to assess motor function (Kopp et al., 1997). The AMAT consists of 13 upper limb tasks of activities of daily living that depend upon shoulder/elbow function and is measured according to the time to complete each of the tasks.

\section{5. fMRI activation analysis. Measures of voxel count and percent signal intensity change}

For each ROI, we calculated the average active voxel count and also the average signal intensity (SI) of the active cluster.

fMRI activation was calculated as follows. For the combined 34 subjects (11 controls and 23 stroke) we computed an average signal intensity map, using an independent sample $t$-test to identify voxels in which there was a signal intensity difference between the rest and the move conditions $(p<0.05)$, using standard correction for multiple comparisons(Benjamini and Hochberg 1995; Genovese et al., 2002). The resultant threshold $p$-value $=0.00062$ was then applied in order to determine brain activation in individual subjects, contrasting rest versus move conditions. An average control activation map was generated for the control subjects.

Percent signal intensity (SI) during the motor task, for each subject, per voxel was calculated as follows: $\% \quad \mathrm{SI}_{\mathrm{v}}=100 *(\operatorname{avgIntM}$-avgIntR $) / \mathrm{avgIntR}, \quad$ where, $\mathrm{SI}_{\mathrm{v}}=$ signal intensity per given voxel, avgInt $M=$ average signal intensity of the given voxel across all motor task scans, avgInt $R=$ average intensity across all rest scans.

The average SI map for control subjects was calculated by voxel-based averaging of SI values across control subjects.

\section{6. fMRI measure of laterality index}

Laterality index was calculated for comparison of the task-related activation in hemispheres contralateral vs ipsilateral to the moving arm (Marshall et al., 2000). LI's were defined for each ROI as $(\mathrm{C}-\mathrm{I}) /(\mathrm{C}+\mathrm{I})$, where $\mathrm{C}$ was the number of active voxels in the contralateral hemisphere and I was that for the ipsilateral hemisphere (Marshall et al., 2000).

\section{7. fMRI measure of euclidean distance from average control brain map centroid}

Centroid was defined and calculated according to published methods (Fesl et al., 2008), and has been used by others studying brain function after stroke (Carey et al., 2006; Jaillard et al., 2005). Centroid was defined as the voxel location within the 3-dimensional space of a given ROI that was both of greatest intensity of activation and most centrally located within the cluster of most intensely activated voxels in that given ROI (Fesl et al., 2008). These two criteria of location and intensity were weighted in order to derive the voxel location taking both of these criteria into account (Fesl et al., 2008). The centroid was first determined for an average map across the control group using the average SI map generated from the eleven controls. The centroid was also calculated within each control subject and within each stroke subject, within each ROI.

Calculation of the centroid coordinates in each ROI was percent SI-weighted. 


$$
\begin{aligned}
C_{x} & =\frac{\sum_{i=1}^{n} \% S I_{i} x_{i}}{n} \\
C_{y} & =\frac{\sum_{i=1}^{n} \% S I_{i} y_{i}}{n} \\
C_{z} & =\frac{\sum_{i=1}^{n} \% S I_{i} z_{i}}{n}
\end{aligned}
$$

The percent SI-weighted $(\% \mathrm{SI})$ coordinates were calculated as follows:

Where,

$\mathrm{C}=$ centroid voxel coordinate location;

$\mathrm{x}, \mathrm{y}$, and $\mathrm{z}=$ location coordinates;

$\% \mathrm{SI}_{\mathrm{i}}=$ the percent difference intensity at the $\mathrm{i}^{\mathrm{th}}$ voxel.

Within each ROI, the Euclidean distance was calculated between the location of the average control map centroid and the centroid for each individual subject (control and stroke).

\subsection{Statistical analysis}

For stroke versus control groups, we employed the Mann-Whitney U test for non-normal distributions. Second, within each ROI, Spearman correlation analysis was conducted between AMAT score and each of the fMRI measures: voxel count, signal intensity (SI), and distance from the average control centroid. Third, in order to compare our results based on MNI, with the published reports of some others, we transformed our data from MNI to Talaraich coordinates (Lancaster et al., 2007). Fourth, for the centroid variable, descriptive statistics were generated to describe the number of stroke subject centroids either within or outside the range of the location of the centroids for the control subjects, and comparisons were conducted for stroke versus control. For the comparisons of stroke versus control, correction for multiple tests was conducted using the standard Bonferroni-Holmes method (Aickin and Gensler, 1996; Darling et al., 2009), and significant $\mathrm{p}$ values after correction are indicated in Tables 3 and 4 with an asterisk.

\section{Results}

\subsection{Subjects}

Subject characteristics are provided in Table 1 . According to the upper limb Fugl-Meyer coordina- tion scale, 2 subjects were moderately impaired (FM range, 30-49) and 21 were severely impaired (FM range, 10-29; (Duncan et al., 1983; Fugl-Meyer et al., 1975)). Average AMAT score for the stroke cohort was $1636.63 \pm 668.42$ seconds.

\subsection{Control subjects; characterization of brain activation during the reach task}

Results showed that healthy adults activated bilateral primary and secondary cortical motor-sensory regions during the functional reach task. The pattern of average control group activation is shown in Fig. 2 (panel A), and in Table 2. For controls, the activated brain volume was greater in the hemisphere contralateral to the moving limb, as expected, in all ROIs shown in Table 2, column D. That is, for each of the ROIs listed in Table 2, brain activation (column D) was 1.8 to 12 times greater for the hemisphere contralateral to the moving arm versus the ipsilateral hemisphere.

\subsection{Comparison of cortical and sub-cortical stroke}

From the comparison analysis of subjects with cortical versus subcortical lesions, we found no difference between those two groups in any ROIs according to

Table 1

Subject characteristics

\begin{tabular}{lcc}
\hline & $\begin{array}{c}\text { Stroke } \\
\text { subjects } \\
n=23\end{array}$ & $\begin{array}{c}\text { Control } \\
\text { subjects } \\
n=11\end{array}$ \\
\hline Age in years, mean (std dev) & $56.3(12.8)$ & $54.4(12.9)$ \\
Female (\%) & $41 \%$ & $54 \%$ \\
Stroke hemisphere (\% Left) & $55 \%$ & \\
Stroke Type (\% ischemic) & $88.6 \%$ & \\
Right dominant $n(\%)$ & $16(70 \%)$ & \\
Dominant arm affected $n(\%)$ & $15(65 \%)$ & \\
Lesion location $n(\%)$ & & \\
BG/IC & $7(30 \%)$ & \\
Pons & $2(8.6 \%)$ & \\
Frontal lobe & $1(2.3 \%)$ & \\
Frontal/parietal lobes & $3(13 \%)$ & \\
Frontal lobe/BG/IC & $3(13 \%)$ & \\
Frontal/parietal lobes/BG/IC & $5(21.7 \%)$ & \\
Frontal/parietal/temporal lobes/BG/IC & $2(8.6 \%)$ & \\
Years since stroke, mean (SD) & $1.8(1.1)$ \\
Medical history & & \\
DM & $17.4 \%$ \\
HTN & $52.2 \%$ \\
Heart disease & $21.7 \%$ \\
Smoking & $56.5 \%$ \\
\hline
\end{tabular}




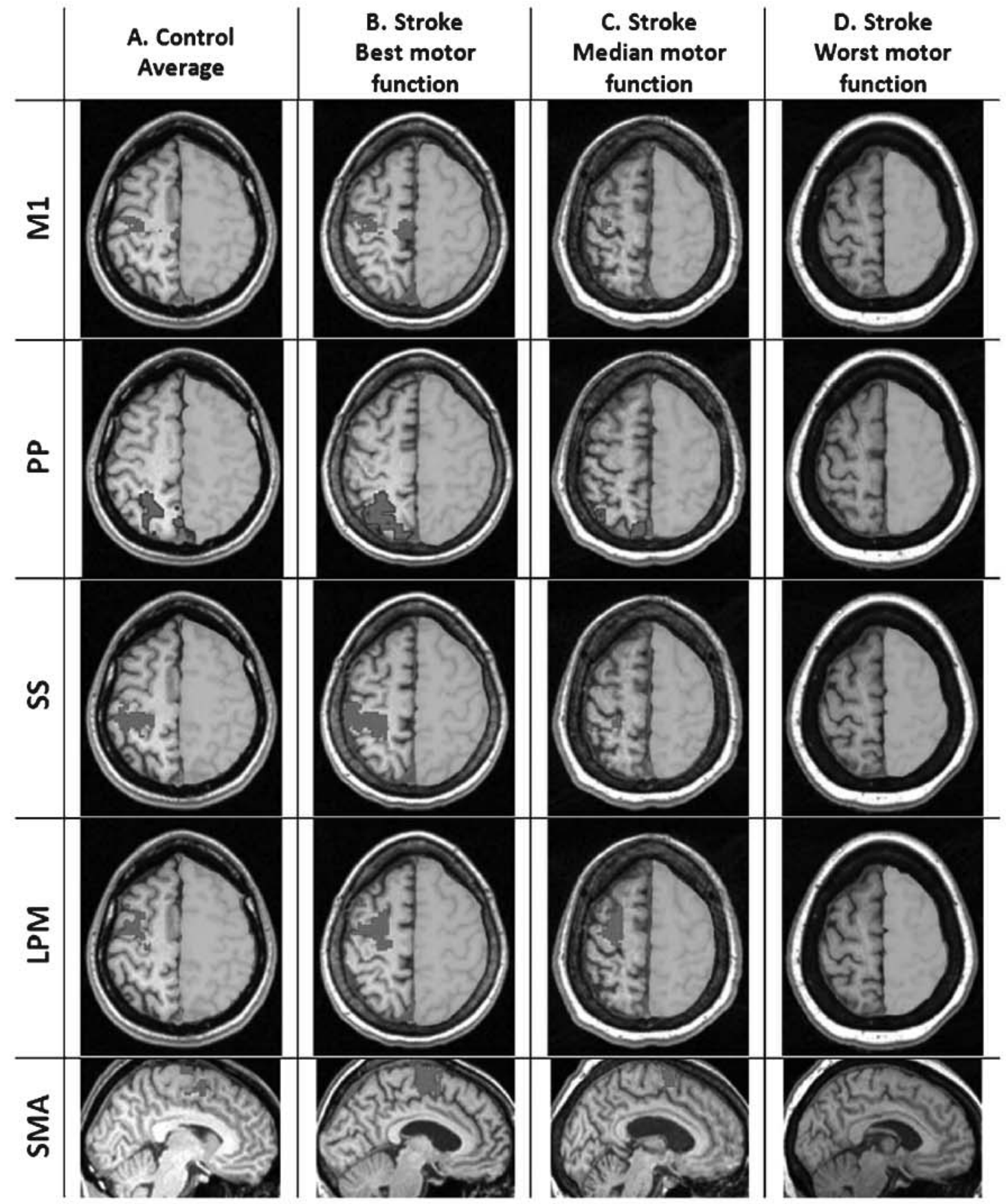

Fig. 2. Four case examples of ipsilesional hemisphere activations for healthy adult (A), stroke good motor function (B.), stroke median motor function (C), and stroke poor motor function (D). M1 = primary motor cortex; PP = posterior parietal area; $\mathrm{SS}=$ somatosensory area; LPM = lateral premotor area; SMA = supplementary motor area; Light Gray area - right hemisphere light gray shading indicates illustration for completeness only (no activations shown). 
Table 2

Average activation map for healthy controls during a shoulder/elbow reach task

\begin{tabular}{|c|c|c|c|c|c|}
\hline \multirow[t]{2}{*}{ A. ROIs } & \multirow[t]{2}{*}{ B. Hemi sphere } & \multicolumn{3}{|c|}{$\begin{array}{l}\text { C. Centroid } \\
\text { Coordinate }\end{array}$} & \multirow[t]{2}{*}{$\begin{array}{l}\text { D. Voxel } \\
\text { count }\end{array}$} \\
\hline & & $\mathrm{X}$ & $\mathrm{Y}$ & $\mathrm{Z}$ & \\
\hline \multirow[t]{2}{*}{ M1 } & $\mathrm{C}$ & -31 & -26 & 61 & 659 \\
\hline & I & 12 & -30 & 68 & 138 \\
\hline \multirow[t]{2}{*}{ SS } & $\mathrm{C}$ & -42 & -32 & 54 & 1530 \\
\hline & I & 37 & -39 & 61 & 515 \\
\hline \multirow[t]{2}{*}{ LPM } & C & -31 & -11 & 61 & 1067 \\
\hline & I & 21 & -3 & 70 & 88 \\
\hline \multirow[t]{2}{*}{ SMA } & $\mathrm{C}$ & -10 & -4 & 69 & 433 \\
\hline & I & 9 & -2 & 66 & 246 \\
\hline \multirow[t]{2}{*}{ PP } & $\mathrm{C}$ & -14 & -58 & 63 & 1454 \\
\hline & I & 17 & -59 & 63 & 1336 \\
\hline \multirow[t]{2}{*}{ Hemi sphere } & C & -33 & -25 & 47 & 10304 \\
\hline & I & 32 & -37 & 43 & 7360 \\
\hline
\end{tabular}

$\mathrm{C}=$ hemisphere contralateral to the working arm. $\mathrm{I}=$ hemisphere ipsilateral to the working arm. M1=primary motor cortex. $\mathrm{PP}=$ posterior parietal area. $\mathrm{SS}=$ somatosensory area. $\mathrm{LPM}=$ lateral premotor area.

both volume of brain activation or intensity of activation $(p>0.2)$.

\subsection{Comparison of stroke and control according to laterality index}

For the control group, the overall Laterality Index (LI) was significantly greater than for the stroke group $(p=0.001)$. Though not significant after correction for multiple tests, there was a trend toward significance in two ROIs: LPM and SMA ( $p=0.03 ; p=0.03$, respectively, Table 3). These results, together, indicate that for the relative activation in the hemisphere contralateral (lesion side) to the working arm versus the ipsilateral hemisphere, the control group had a greater relative difference in hemisphere activation versus the stroke group (greater laterality, favoring greater activation in the hemisphere contralateral to the working limb).

\subsection{Comparison of stroke and control according to the distance between individual subject centroid and the average control map centroid}

For all five ROIs, Table 4 shows a comparison of the control group versus the stroke group according to the linear distance between individual subject centroid and the centroid for the average control map centroid. The difference between stroke and control was statistically significant in the ipsilesional hemisphere (contralateral to the working arm) for M1 and SS (Table 4, column E);

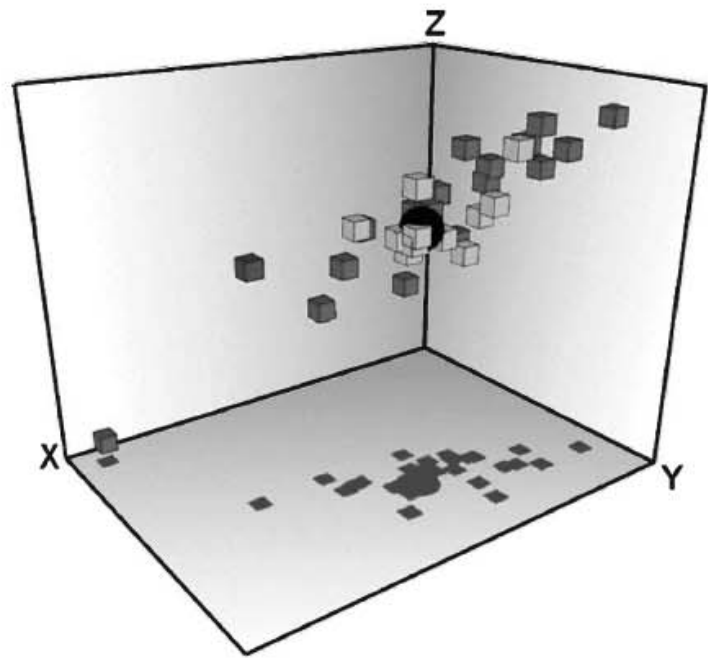

Fig. 3. M1 Centroids (Ipsilesional Hemisphere) for all study subjects (healthy control (light shade (green) cubes) and stroke (dark shade (red) cubes) subjects) in relationship to the average control group centroid (black sphere).

though not reaching significance after correction for multiple tests, there were trend values for LPM, SMA, and PP (Table 4, column E). There was a different pattern of activation for the contralesional hemisphere (ipsilateral to the tested arm) ROIs; that is, there was a significant difference between stroke and control for the SS and LPM regions ( $p<0.006$; column E).

Figure 3 shows a graphic illustration of the centroid data distribution for control group (light shade (green) cubes) and stroke group (dark shade (red) cubes), for the M1 ROI, as well as the average control map centroid (black cube) for that M1 region of interest. Though statistical analysis results are described in the previous paragraph, this illustration of the centroids in the M1 ROI, shows that the centroid locations for seven stroke survivors were within the area circumscribed by all the centroids of the control group; whereas, centroid locations for 13 of the stroke survivors were beyond the area circumscribed by the centroid locations for the control subjects.

\subsection{Correlation of brain activation measures with motor functional task performance}

\subsubsection{Correlation of extent of brain activation with motor function task performance}

There was a good correlation (Portney and Watkins, 2000), which was statistically significant, between 
Table 3

Comparison of stroke versus control according to the laterality index (LI)

\begin{tabular}{lccc}
\hline ROIs & Control mean LI (std) & Stroke mean LI (std) & $p$ value \\
\hline M1 & $0.55(0.32)$ & $0.32(0.49)$ & 0.17 \\
SS & $0.34(0.44)$ & $0.15(0.45)$ & 0.36 \\
LPM & $0.47(0.29)$ & $0.12(0.48)$ & 0.03 \\
SMA & $0.30(0.35)$ & $0.03(0.46)$ & 0.03 \\
PP & $0.21(0.31)$ & $-0.17(0.61)$ & 0.06 \\
LI for the Combined ROIs & $0.37(0.36)$ & $0.06(0.53)$ & $<0.001^{*}$ \\
\hline
\end{tabular}

$\mathrm{M} 1=$ primary motor cortex. $\mathrm{PP}=$ posterior parietal area. $\mathrm{SS}=$ somatosensory area. $\mathrm{LPM}=$ lateral premotor area. *significant $p$ value after corrections for multiple tests.

Table 4

Comparison of stroke vs. control according to the distance from the COM in the average control brain map

\begin{tabular}{lcccc}
\hline A.ROI & B. Side & \multicolumn{3}{c}{ Distance from Average Control Brain Map COM } \\
\cline { 3 - 5 } & & C. Control mean (SD) & D. Stroke mean (SD) & E. $p$ value \\
\hline M1 & C & $5.21(3.78)$ & $12.02(9.4)$ & $0.007^{*}$ \\
& I & $9.13(4.91)$ & $17.58(15.33)$ & 0.1 \\
SS & C & $4.23(2.69)$ & $10.33(7.64)$ & $0.01^{*}$ \\
& I & $8.73(12.09)$ & $19.40(12.95)$ & $0.006^{*}$ \\
LPM & C & $6.53(4.85)$ & $14.47(13.0)$ & 0.03 \\
& I & $5.30(4.48)$ & $27.12(10.87)$ & $0.0003^{*}$ \\
SMA & C & $4.21(1.80)$ & $7.39(4.56)$ & 0.04 \\
& I & $5.37(3.03)$ & $5.17(2.77)$ & 0.9 \\
PP & C & $5.56(2.85)$ & $10.51(6.63)$ & 0.026 \\
& I & $6.17(2.27)$ & $9.24(7.08)$ & 0.185 \\
\hline
\end{tabular}

$\mathrm{C}=$ hemisphere contralateral to the working arm (lesioned hemisphere). $\mathrm{I}=$ hemisphere ipsilateral to the working arm (non-lesioned hemisphere). $\mathrm{M} 1=$ primary motor cortex. $\mathrm{PP}=$ posterior parietal area $\mathrm{SS}=$ somatosensory area. $\mathrm{LPM}=$ lateral premotor area. *significant after correction for multiple tests.

voxel count and motor functional task performance (AMAT measure of upper limb function) in the primary and the secondary motor-sensory regions (Table 5, column C) of the hemisphere contralateral to the working arm (lesioned hemisphere; correlations ranged, $r=0.51$ to $r=0.73$; Fig. 4). Similar results were found in the non-lesioned hemisphere (ipsilateral to the working arm), but for only two ROIs, M1 and SMA (non-lesioned; $r=0.47$ and 0.43 , respectively). This finding indicates that for this cohort of chronic severely impaired stroke survivors, greater extent of brain activation in both primary and secondary motor-sensory regions was operative for those with better motor functional task performance.

Figure 2 provides three examples of stroke survivors and the average for the control group, with each showing ipsilesional hemisphere activation (contralesional hemisphere is shaded to indicate that only the ipsilesional hemisphere activations are shown here for these examples in Fig. 2). These single subject examples are provided to illustrate the difference in brain
Table 5

Correlation of functional task performance with measures of brain function in stroke subjects

\begin{tabular}{lccc}
\hline A. ROI & B. Hemi sphere & $\begin{array}{c}\text { C. Voxel Count } \\
\text { Correlation with } \\
\text { Functional } \\
\text { Performance }\end{array}$ & $\begin{array}{c}\text { D. Signal Intensity } \\
\text { Correlation with } \\
\text { Functional } \\
\text { Performance }\end{array}$ \\
\hline M1 & C & $0.525^{*}$ & $0.585 \ddagger$ \\
SS & I & $0.473^{*}$ & $0.747 \ddagger$ \\
& C & $0.537^{*}$ & $0.639 \ddagger$ \\
LPM & I & $0.522^{*}$ & \\
& C & $0.513^{*}$ & \\
SMA & I & $0.425^{*}$ & $0.656 \ddagger$ \\
PP & C & $0.732 *$ & \\
\hline & C & & \\
\hline
\end{tabular}

$\mathrm{C}=$ hemisphere contralateral to the working arm (lesioned hemisphere). $\mathrm{I}=$ hemisphere ipsilateral to the working arm (non-lesioned hemisphere). $\mathrm{M} 1=$ primary motor cortex. $\mathrm{PP}=$ posterior parietal area. $\mathrm{SS}=$ somatosensory area. LPM $=$ lateral premotor area. ${ }^{*}$ Correlations of $>0.4$ and with $p<0.05, \ddagger$ Correlations of $>0.4$ and with $p<0.01$. 

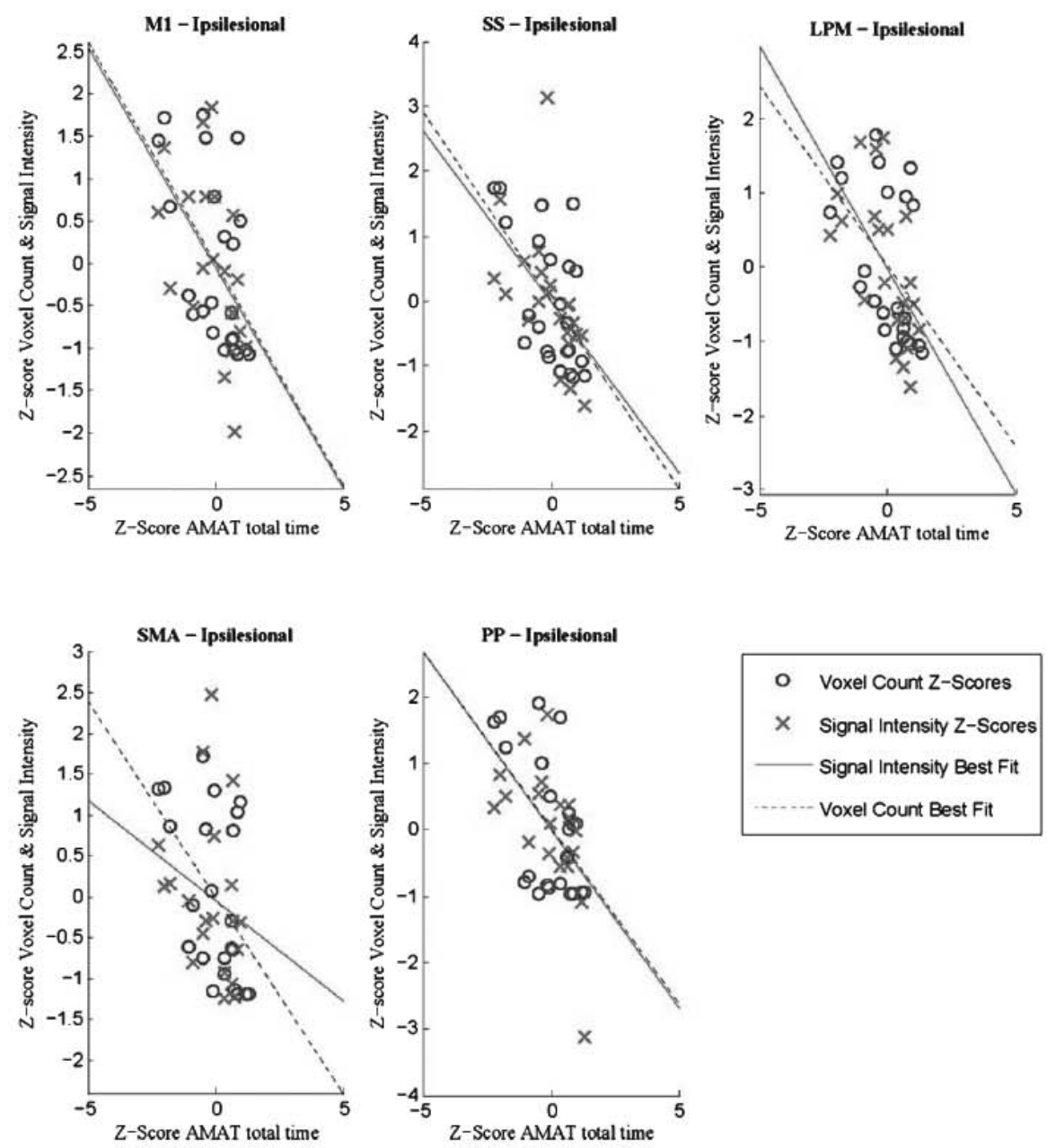

Fig. 4. Correlations between AMAT and both extent of activation (voxel count) and activation intensity (signal intensity) in five ROIs in the ipsilesional hemisphere (contralateral to the working arm). Each value is expressed as a z-score.

activation for severely and more mildly impaired stroke survivors.

\subsubsection{Signal intensity correlation with motor function task performance}

There was a good to excellent correlation (Portney and Watkins, 2000), which was statistically significant, between brain signal intensity and functional task performance in the lesioned hemisphere (contralateral to the working arm), as follows: primary motor $(r=0.585$, $p=0.007)$, somatosensory $(r=0.747, p<0.001)$, lateral premotor $(r=0.639, p=0.002)$ and posterior parietal regions ( $r=0.656, p=0.002)$ (Table 5 , column D; and Fig. 4). These findings indicate that there was a higher signal intensity associated with better motor function in the lesioned hemisphere (contralateral to the working arm) for primary and secondary motorsensory regions during task execution.

\section{Discussion}

This study contributes to the literature in two ways. First, to our knowledge, this is the first study that identifies the specific patterns of brain activation during the basic components of functional reach, shoulder flexion/elbow extension in moderately to severely impaired, chronic stroke survivors. Second, 
Table 6

Comparison of MNI and Talairach (tal) coordinates for the average activation map for healthy controls during a shoulder/elbow reach task

\begin{tabular}{lcccc}
\hline ROIs & Hemi sphere & \multicolumn{3}{c}{ Centroid Coordinate } \\
\cline { 3 - 5 } & & X-MNI (X-tal) & Y-MNI (X-tal) & Z-MNI (Z-tal) \\
\hline M1 & C & $-31(-31)$ & $-26(-31)$ & $61(56)$ \\
& I & $12(9)$ & $-30(36)$ & $68(62)$ \\
SS & C & $-42(-41)$ & $-32(-36)$ & $54(49)$ \\
& I & $37(33)$ & $-39(-43)$ & $61(56)$ \\
LPM & C & $-31(30)$ & $-11(-17)$ & $61(57)$ \\
& I & $21(17)$ & $-3(-10)$ & $70(66)$ \\
SMA & C & $-10(-11)$ & $-4(-11)$ & $69(65)$ \\
& I & $9(6)$ & $-2(-9)$ & $66(63)$ \\
PP & C & $-14(-15)$ & $-58(-61)$ & $63(55)$ \\
& I & $17(14)$ & $-59(-63)$ & $63(55)$ \\
\hline
\end{tabular}

$\mathrm{C}=$ hemisphere contralateral to the working arm (lesioned hemisphere). $\mathrm{I}=$ hemisphere ipsilateral to the working arm (non-lesioned hemisphere). $\mathrm{M} 1=$ primary motor cortex. $\mathrm{PP}=$ posterior parietal area. $\mathrm{SS}=$ somatosensory area $\mathrm{LPM}=$ lateral premotor area.

we identified a relationship between greater volume and intensity of brain activation, and higher functional task performance.

\subsection{Location of brain control for a functional reach task of shoulder flexion and elbow extension}

Our results showed activation in five ROIs during functional reach, composed of shoulder flexion and elbow extension. Table 2 provides the results from the current study according to centroid coordinates using the MNI template; Table 6 provides a side-by-side report of the MNI centroid coordinates, as well as the coordinates converted into Talairach, for the purpose of comparison of our findings with the work of others.

There have been only a few prior neuroimaging studies addressing shoulder movements in chronic stroke survivors, and these focused on a single movement of the shoulder. Our results for centroid location are consistent with the results of others who studied a simple shoulder rotation task; that is, brain activation for more proximal muscles is located more dorsally. Specifically, we found that in the M1 ROI, the y coordinate for reach was ' $y$-tal $=-26$ ' which was very similar to the y-tal coordinate reported by others for the shoulder external rotation movement $(\mathrm{y}-\mathrm{tal}=-27$; primary sensorimotor cortex (precentral plus postcentral gyrus from vertex to $7 \mathrm{~mm}$ above Sylvian fissure (Cramer and Crafton, 2006)). This is a reasonable similarity, given that the $y$ coordinate determines a ventral/dorsal location on the motor homunculus.
Unlike the simple shoulder movement task studied by others, the reach task includes elbow extension and shoulder flexion against gravity. Therefore, in contrast to others who studied only a simple humerus rotation movement (Cramer and Crafton, 2006), we found that the ' $x$ ' centroid coordinate for the reach task, which involves both shoulder and elbow joints, was more lateral, as expected (Kandel et al., 2000), than that of the single joint shoulder rotation movement. This greater laterality was reflected by our findings for the $\mathrm{x}$-tal coordinate in the M1 ROI ( $\mathrm{x}$-tal $=31$; hemisphere contralateral to moving arm), which was more lateral than the $x$-tal $=22$ for the simple shoulder rotation task (Cramer and Crafton, 2006). Our average control M1 centroid coordinates (contralateral to moving arm) represent a location within the dorsal curvature of the precentral gyrus, an expected site for primary motor region control of a shoulder flexion and elbow extension reach movement (Kandel et al., 2000).

It is well-known that afferent feedback plays an important part in guiding normal active movement, and that afferent feedback during passive movement can generate brain activations measured by fMRI (Nelles et al., 1999). In the current study, we investigated active movement, which inherently includes both efferent and afferent neural activity. In future work, it may be helpful to study afferent (passive movement), as differentiated from active movement. In this paper, we have referred to brain activations as "task-related", meant to include both efferent and afferent activity that is captured by fMRI.

Functional connectivity measures are emerging as an important indication of the complexity of the brain control of the motor system (Grefkes and Fink, 2014), which is composed of interacting cortical and subcortical areas. The integrity of regions of activity and their connections are critical to normal control of motor function; therefore it is important to identify the locus of activations as well as their connections, for particular motor functions. Our findings for stroke are consistent with studies on functional reaching in healthy adults. That is, for some ROIs there is a relationship between motor function and brain activation in both hemispheres during a unilateral movement. For example, according to studies in healthy control subjects (Gallivan et al., 2013; Bernier et al., 2012; Kertzman et al., 1997), it is reasonable to assume that activation in both PP and SMA regions represent movement planning-related activation, and that bilateral hemisphere relationships are important (bilateral 
M1 (Gallivan et al., 2013) and bilateral SMA (Kertzman et al., 1997). Emerging evidence is indicating that re-organization of these networks may contribute to recovery of motor control (Grefkes and Fink, 2014; Jiang et al., 2013). The results of the current study provide a first step in that direction by identifying the location of brain activations in an array of ROI's serving motor control and their brain function impairments after stroke for functional reach. These results can be utilized in future work to target specific ROIs and their connections so that treatment can be more accurately targeted to modulate connectivity between these regions (Jiang et al., 2013; Grefkes and Fink, 2014).

\subsection{Comparison of laterality index in healthy adults and stroke survivors}

The Laterality Index has been used in post-stroke fMRI studies to identify neuroplastic changes after stroke (Marshall et al., 2000). The current work provides three findings of note for the reach task. First, we found greater laterality in controls versus stroke. Our LI values for the shoulder/elbow reach task for healthy adults ranged 0.08 to 0.55 ; and the LI values for stroke survivors were lower than for healthy adults and ranged -0.08 to 0.32 . The stroke group demonstrated less lateralization than healthy controls, in the SMA and LPM regions. These findings indicate that for healthy adults in comparison to stroke survivors, there was relatively greater activation of the hemisphere contralateral to the working limb versus the activation in the hemisphere ipsilateral to the working limb.

Second, in stroke survivors, we found an LI that indicated a greater activation in the hemisphere contralateral to the working limb (lesioned hemisphere) versus the hemisphere ipsilateral to the working limb (non-lesioned hemisphere). These results are consistent with that of Marshall and colleagues (Marshall et al., 2000), who reported that brain activation was more highly lateralized to the lesioned hemisphere (contralateral to the working arm) versus healthy adults, for the finger/thumb opposition task ( $\mathrm{LI}=0.32$ (range -0.2 to 0.8 ) at 3-6 months after stroke). Others reported an LI for a simple humeral rotation movement $(\mathrm{LI}=0.8)$ and a finger tap movement $(\mathrm{LI}=0.79$ (Cramer and Crafton, 2006)) for stroke survivors over 4 months after stroke.

Third, the low laterality index for healthy controls could reflect the bihemispheric nature of shoul- der/elbow movement control; since many tasks are bilateral, a unilateral single limb right arm reach (driven by the left hemisphere) might require activation of the right hemisphere to inhibit the habitual left limb bilateral functional movements often demanded (Chen et al., 1997; Avanzino et al., 2011). In this example, the right hemisphere might also inhibit the antagonist muscles (the large shoulder extensors and elbow flexors) which have much greater force-generating capability than those muscle groups employed in the reach task. Furthermore, a role of ipsilateral motor control appears to be important for the proximal limb muscles; this phenomenon was demonstrated in the studies that described that ipsilateral motor evoked response can be induced with stimulation of the cortical regions for the proximal muscle groups (Wassermann et al., 1994; Strutton et al., 2004).

Fourth, we found that for the functional reach task, there was a lower LI than for more simple or distal limb tasks, studied by others. This is reasonable, considering a number of factors. The shoulder/elbow task requires control of more than one joint and requires lifting the arm against gravity. Additionally, activation of shoulder muscles can engage scapular stabilizers and other muscles of the shoulder capsule mechanism, as well as torso stabilizers. Also, in comparison to a single movement at a single joint, the reach movement of shoulder flexion/elbow extension is an 'out-of-synergy' movement, requiring the neural system to execute this more highly coordinated motor task, which is often impaired in stroke survivors (Carr and Shepherd, 2011).

\subsection{Correlation between brain activation and functional motor tasks}

The results of the current work showed a strong correlation between complex motor functional task performance and two measures of brain activation (extent of activation and intensity of activation $(r=0.5$ to 0.7 ) for primary and secondary motor and sensory control regions of the brain. The confluence of these findings supports the conclusion that for severely impaired chronic stroke survivors, greater activation in the motor and sensory control system of the ipsilesional hemisphere (contralateral to working arm) is associated with better functional task performance.

Our work extends the literature in two ways: a more complex shoulder/elbow reach task was studied during brain measurement; and the function measure, to which we correlated brain function, is composed of 
actual functional tasks (i.e., not simple movement tests, such as box and blocks, Fugl-Meyer joint movement test). The more complex reach task, used in the current study to acquire brain function data, provides information beyond that of prior studies which used motor tasks inside the scanner that were simple single joint movements or distal tasks (e.g., (Cramer and Crafton 2006; Feydy et al., 2002)). And, in the current work, for purposes of correlation of brain function with everyday motor tasks, we used the AMAT, which is based on 13 complex functional tasks. Others reported correlations of brain activation measures with motor function, but in those studies, the motor function measures were at the impairment level (pegboard test, Fugl-Meyer coordination test, measures of strength, finger-tapping rate) (Askim et al., 2009; Calautti et al., 2010; Cramer et al., 2002; Fujii and Nakada, 2003; Jang et al., 2004), rather than at the functional task level. Additionally, in prior work, others reported correlations between scanner tasks and motor tasks, and were limited to a single MRI measure of brain activation (Askim et al., 2009, Calautti et al., 2010; Cramer et al., 2002; Fujii and Nakada, 2003; Jang et al., 2004; Kokotilo et al., 2010; Marshall et al., 2000; Ward et al., 2003; Zemke et al., 2003); whereas, in the current work, we report the confluence of results from two measures of brain function and their respective correlation with a measure of complex functional tasks. Most prior reports were limited to the primary sensorimotor area, but reported as we do, that better motor function was associated with greater activation in ipsilesional primary sensorimotor areas (Calautti et al., 2010; Carey et al., 2005; Jang et al., 2004; Marshall et al., 2000; Zemke et al., 2003), and with either increased (Fujii and Nakada, 2003) or decreased (Cramer and Crafton, 2006) activation in contralesional primary sensorimotor area. In contrast to these studies, our study extends the literature by quantifying an array of active task-related brain regions, suggesting a network of cortical sensorymotor regions relevant to skilled motor function.

For the most part, our results are consistent with the work of others. Only a few studies were not consistent with the main body of work of others and our own work. These few studies found no correlation between brain activation and physical movement capability (Feydy et al., 2002; Loubinoux et al., 2007; Small et al., 2002); but of note, those studies used a different approach for correlation data analysis (Loubinoux et al., 2003) or smaller sample size and a different statistical approach (Feydy et al., 2002; Small et al., 2002).
Correlation values ranged from 0.5 to 0.7 . Though correlations were good, subject variability was exhibited (Fig. 4). There are a number of variables that could have an effect on the relationship between brain activation measures and functional capability, as follows: volume of lesion (Darling et al., 2009; Cramer et al., 2007; Rickards et al., 2012), type of stroke and number of motor control regions involved (Mestriner et al., 2013), degree of white matter involvement (Hedna et al., 2013), and functionality of intracortical network connections (Jiang et al., 2013; Grefkes and Fink, 2014). In future studies of a larger sample size, investigation of each of these variables will be required in order to more fully understand the variables affecting the relationship of brain activation levels for each motor control region and functional motor control.

\subsection{Research and clinical implications}

Current clinical practice is not guided by accurate knowledge of the relationship of brain impairment and arm motor dysfunction, particularly for the functional reach, basic components of shoulder flexion/elbow extension. The results of this study provide a description of brain activation in an array of ROIs which normally serve motor control of the reach component movements, and in which brain impairment is located after stroke; three types of brain measures are provided; volume of brain activation, intensity of brain activation, and centroid location of brain activation during this task; with the identification of these benchmark measures of impaired brain function, future work can then attempt to more accurately target these brain function impairments in treatment methods. The study findings indicate that greater brain activation was associated with higher functional level for severely impaired, chronic stroke survivors. Therefore, training could include methods for enhancing brain activation in these identified ROIs. One example of using these fMRI findings could be to employ real time fMRI (rtfMRI) neural feedback treatment to target these identified ROIs and utilize up-regulation training of brain activity to more accurately target brain training for shoulder flexion/elbow extension training for motor recovery; this kind of rtfMRI targeted treatment is currently emerging in research reports and has been attempted for elbow flexion (Sitaram et al., 2012), but not yet for shoulder flexion/elbow extension reach movement, which is a more difficult measurement problem. 


\section{Summary}

This study provides new information for moderately to severely motor impaired, chronic stroke survivors, with regard to brain activation patterns during the basic components of function reach, shoulder flexion/elbow extension; brain activation patterns for stroke were significantly different from healthy controls, according to measures of volume of activation, laterality of activation, and centroid location, in a number of ROIs.

Results showed that two brain activation measures (volume of activation and intensity of activation) during shoulder flexion/elbow extension reach were correlated with a measure of complex functional motor tasks, across primary and secondary regions responsible for motor control. Overall, a greater extent and intensity of brain activation correlated with better motor function. Taken together, these measures characterizing impairment of brain function can be used to more accurately target motor recovery interventions. For example, our results suggest that recovery of proximal arm motor control may be enhanced through bi-hemispheric engagement, which is in contrast to training for recovery of distal arm function. Moreover, for shoulder/elbow motor control recovery, it may prove productive, through neural feedback training or direct brain stimulation, to enhance activation for the regions identified here, for more focused treatment. Generating and testing innovative methods to induce engagement of the appropriate brain regions to recover control of shoulder/elbow motor function may be a fruitful pathway to improving neurorehabilitation after stroke.

\section{Acknowledgement}

The study was supported by grants from the Department of Veterans Affairs Rehabilitation Research and Development Service (\#B3709R, \#B9024S and \#B6636W).

\section{References}

Acuna, M., Amasay, T., \& Karduna, A.R. (2010). The reliability of side to side measurements of upper extremity activity levels in healthy subjects. BMC Musculoskelet Disord, 11, 168.

Aickin, M., \& Gensler, H. (1996). Adjusting for multiple testing when reporting research results: The Bonferroni vs Holm methods. Am J Public Health, 86(5), 726-728.
Askim, T., Indredavik, B., Vangberg, T., \& Haberg, A. (2009) Motor network changes associated with successful motor skill relearning after acute ischemic stroke: A longitudinal functional magnetic resonance imaging study. Neurorehabil Neural Repair, 23(3), 295-304.

Avanzino, L., Bassolino, M., Pozzo, T., \& Bove, M. (2011). Usedependent hemispheric balance. J Neurosci, 31(9), 3423-3428.

Benjamini, Y., \& Hochberg, Y. (1995). Controlling the false discovery rate: A practical and powerful approach to multiple testing. $J$ R Stat Soc Ser, 57, 289-300.

Bernier, P.M., Cieslak, M., \& Grafton, S.T. (2012). Effector selection precedes reach planning in the dorsal parietofrontal cortex. $J$ Neurophysiol, 108(1), 57-68.

Calautti, C., Jones, P.S., Naccarato, M., Sharma, N., Day, D.J., Bullmore, E.T., Warburton, E.A., \& Baron, J.C. (2010). The relationship between motor deficit and primary motor cortex hemispheric activation balance after stroke: Longitudinal fMRI study. J Neurol Neurosurg Psychiatry, 81(7), 788-792.

Carey, L.M., Abbott, D.F., Egan, G.F., Bernhardt, J., \& Donnan, G.A. (2005). Motor impairment and recovery in the upper limb after stroke: Behavioral and neuroanatomical correlates. Stroke, 36(3), 625-629.

Carey, L.M., Abbott, D.F., Egan, G.F., O’Keefe, G.J., Jackson, G.D., Bernhardt, J., \& Donnan, G.A. (2006). Evolution of brain activation with good and poor motor recovery after stroke. Neurorehabil Neural Repair, 20(1), 24-41.

Carod-Artal, F.J., \& Egido, J.A. (2009). Quality of life after stroke: The importance of a good recovery. Cerebrovasc Dis 27(Supp 1), 204-214.

Carr, J.H., \& Shepherd, R.B. (2011). Enhancing physical activity and brain reorganization after stroke. Neurol Res Int 2011, 515-938.

Cavina-Pratesi, C., Monaco, S., Fattori, P., Galletti, C., McAdam, T.D., Quinlan, D.J., Goodale, M.A., \& Culham, J.C. (2010) Functional magnetic resonance imaging reveals the neural substrates of arm transport and grip formation in reach-to-grasp actions in humans. J Neurosci, 30(31), 10306-10323.

Chen, R., Cohen, L.G., \& Hallett, M. (1997). Role of the ipsilateral motor cortex in voluntary movement. Can J Neurol Sci, 24(4), 284-291.

Crafton, K.R., Mark, A.N., \& Cramer, S.C. (2003). Improved understanding of cortical injury by incorporating measures of functional anatomy. Brain, 126(Pt 7), 1650-1659.

Cramer, S.C., \& Crafton, K.R. (2006). Somatotopy and movement representation sites following cortical stroke. Exp Brain Res, 168(1-2), 25-32.

Cramer, S.C., Parrish, T.B., Levy, R.M., Stebbins, G.T., Ruland, S.D., Lowry, D.W., Trouard, T.P., Squire, S.W., Weinand, M.E., Savage, C.R., Wilkinson, S.B., Juranek, J., Leu, S.Y., \& Himes, D.M. (2007). Predicting functional gains in a stroke trial. Stroke, $38(7), 2108-2114$.

Cramer, S.C., Weisskoff, R.M., Schaechter, J.D., Nelles, G., Foley, M., Finklestein, S.P., \& Rosen, B.R. (2002). Motor cortex activation is related to force of squeezing. Hum Brain Mapp, 16(4), 197-205.

Daly, J.J., Hrovat, K., Pundik, S., Sunshine, J., \& Yue, G. (2008). fMRI methods for proximal upper limb 
joint motor testing and identification of undesired mirror movement after stroke. J Neurosci Methods, 175(1), 133-142.

Darling, W.G., Pizzimenti, M.A., Rotella, D.L., Peterson, C.R., Hynes, S.M., Ge, J., Solon, K., McNeal, D.W., StilwellMorecraft, K.S., \& Morecraft, R.J. (2009). Volumetric effects of motor cortex injury on recovery of dexterous movements. Exp Neurol, 220(1), 90-108.

Dean, H.L., Hagan, M.A., \& Pesaran, B. (2012). Only coherent spiking in posterior parietal cortex coordinates looking and reaching. Neuron, 73(4), 829-841.

Desmurget, M., Grea, H., Grethe, J.S., Prablanc, C., Alexander, G.E., \& Grafton, S.T. (2001). Functional anatomy of nonvisual feedback loops during reaching: A positron emission tomography study. J Neurosci, 21(8), 2919-2928.

Dong, Y., Winstein, C.J., Albistegui-DuBois, R., \& Dobkin, B.H. (2007). Evolution of FMRI activation in the perilesional primary motor cortex and cerebellum with rehabilitation training-related motor gains after stroke: A pilot study. $\mathrm{Neu}$ rorehabil Neural Repair, 21(5), 412-428.

Duncan, P.W., Propst, M., \& Nelson, S.G. (1983). Reliability of the Fugl-Meyer assessment of sensorimotor recovery following cerebrovascular accident. Phys Ther, 63(10), 1606-1610.

Fesl, G., Braun, B., Rau, S., Wiesmann, M., Ruge, M., Bruhns, P., Linn, J., Stephan, T., Ilmberger, J., Tonn, J.C., \& Bruckmann, H. (2008). Is the center of mass (COM) a reliable parameter for the localization of brain function in fMRI? Eur Radiol, 18(5), 1031-1037.

Feydy, A., Carlier, R., Roby-Brami, A., Bussel, B., Cazalis, F., Pierot, L., Burnod, Y., \& Maier, M.A. (2002). Longitudinal study of motor recovery after stroke: Recruitment and focusing of brain activation. Stroke, 33(6), 1610-1617.

Fugl-Meyer, A.R., Jaasko, L., Leyman, I., Olsson, S., \& Steglind, S. (1975). The post-stroke hemiplegic patient. 1. a method for evaluation of physical performance. Scand J Rehabil Med, 7(1), 13-31

Fujii, Y., \& Nakada, T. (2003). Cortical reorganization in patients with subcortical hemiparesis: Neural mechanisms of functional recovery and prognostic implication. J Neurosurg, 98(1), 64-73

Gallivan, J.P., Cavina-Pratesi, C., \& Culham, J.C. (2009) Is that within reach? fMRI reveals that the human superior parietooccipital cortex encodes objects reachable by the hand. $J$ Neurosci, 29(14), 4381-4391.

Gallivan, J.P., McLean, D.A., Flanagan, J.R., \& Culham, J.C. (2013). Where one hand meets the other: Limb-specific and actiondependent movement plans decoded from preparatory signals in single human frontoparietal brain areas. J Neurosci 33(5), 1991-2008.

Genovese, C.R., Lazar, N.A., \& Nichols, T. (2002). Thresholding of statistical maps in functional neuroimaging using the false discovery rate. Neuroimage, $15(4), 870-878$.

Grefkes, C., \& Fink, G.R. (2014). Connectivity-based approaches in stroke and recovery of function. Lancet Neurol, 13(2), 206-216.

Harvey, R.L., Roth, E.J., Heinemann, A.W., Lovell, L.L., McGuire, J.R., \& Diaz, S. (1998). Stroke rehabilitation: Clinical predic- tors of resource utilization. Arch Phys Med Rehabil, 79(11), 1349-1355.

Hedna, V.S., Jain, S., Rabbani, O., \& Nadeau, S.E. (2013). Mechanisms of arm paresis in middle cerebral artery distribution stroke: Pilot study. J Rehabil Res Dev, 50(8), 1113-1122.

Himmelbach, M., Linzenbold, W., \& Ilg, U.J. (2013). Dissociation of reach-related and visual signals in the human superior colliculus. Neuroimage, 82, 61-67.

Jaillard, A., Martin, C.D., Garambois, K., Lebas, J.F., \& Hommel, M. (2005). Vicarious function within the human primary motor cortex? A longitudinal fMRI stroke study. Brain, 128(Pt 5), 1122-1138.

Jang, S.H., Cho, S.H., Kim, Y.H., Kwon, Y.H., Byun, W.M., Lee, S.J., Park, S.M., \& Chang, C.H. (2004). Cortical activation changes associated with motor recovery in patients with precentral knob infarct. Neuroreport, 15(3), 395-399.

Jiang, L., Xu, H., \& Yu, C. (2013). Brain connectivity plasticity in the motor network after ischemic stroke. Neural Plast 2013 , 924192.

Kandel, E., Schwartz, J., Jessell, T., Siegelbaum, S., \& Hudspeth, A.J. (2000). Principles of Neural Science. McGraw-Hill, New York.

Kertzman, C., Schwarz, U., Zeffiro, T.A., \& Hallett, M. (1997). The role of posterior parietal cortex in visually guided reaching movements in humans. Exp Brain Res, 114(1), 170-183.

Kokotilo, K.J., Eng, J.J., McKeown, M.J., \& Boyd, L.A. (2010). Greater activation of secondary motor areas is related to less arm use after stroke. Neurorehabil Neural Repair, 24(1), 78-87.

Konen, C.S., Mruczek, R.E., Montoya, J.L., \& Kastner, S. (2013). Functional organization of human posterior parietal cortex: Grasping- and reaching-related activations relative to topographically organized cortex. J Neurophysiol, 109(12), 2897-2908.

Kopp, B., Kunkel, A., Flor, H., Platz, T., Rose, U., Mauritz, K.H., Gresser, K., McCulloch, K.L., \& Taub, E. (1997). The Arm Motor Ability Test: Reliability, validity, and sensitivity to change of an instrument for assessing disabilities in activities of daily living. Arch Phys Med Rehabil, 78(6), 615-620.

Lancaster, J.L., Tordesillas-Gutierrez, D., Martinez, M., Salinas, F., Evans, A., Zilles, K., Mazziotta, J.C., \& Fox, P.T. (2007). Bias between MNI and Talairach coordinates analyzed using the ICBM-152 brain template. Hum Brain Mapp, 28(11), 11941205 .

Loubinoux, I., Carel, C., Pariente, J., Dechaumont, S., Albucher, J.F., Marque, P., Manelfe, C., \& Chollet, F. (2003). Correlation between cerebral reorganization and motor recovery after subcortical infarcts. Neuroimage, 20(4), 2166-2180.

Loubinoux, I., Dechaumont-Palacin, S., Castel-Lacanal, E., De, B.X., Marque, P., Pariente, J., Albucher, J.F., Berry, I., \& Chollet, F. (2007). Prognostic value of FMRI in recovery of hand function in subcortical stroke patients. Cereb Cortex, 17(12), 2980-2987

Macedo, L.G., \& Magee, D.J. (2008). Differences in range of motion between dominant and nondominant sides of upper and lower extremities. J Manipulative Physiol Ther, 31(8), 577-582. 
Marshall, R.S., Perera, G.M., Lazar, R.M., Krakauer, J.W., Constantine, R.C., \& DeLaPaz, R.L. (2000). Evolution of cortical activation during recovery from corticospinal tract infarction. Stroke, 31(3), 656-661.

Mestriner, R.G., Miguel, P.M., Bagatini, P.B., Saur, L., Boisserand, L.S., Baptista, P.P., Xavier, L.L., \& Netto, C.A. (2013). Behavior outcome after ischemic and hemorrhagic stroke, with similar brain damage, in rats. Behav Brain Res, 244, 82-89.

Nair, D.G., Hutchinson, S., Fregni, F., Alexander, M., PascualLeone, A., \& Schlaug, G. (2007). Imaging correlates of motor recovery from cerebral infarction and their physiological significance in well-recovered patients. Neuroimage, 34(1), 253-263.

Nelles, G., Spiekermann, G., Jueptner, M., Leonhardt, G., Muller, S., Gerhard, H., \& Diener, H.C. (1999). Reorganization of sensory and motor systems in hemiplegic stroke patients. A positron emission tomography study. Stroke, 30(8), 1510-1516.

Picard, N., \& Strick, P.L. (1996). Motor areas of the medial wall: A review of their location and functional activation. Cereb Cortex, $6(3), 342-353$.

Picard, N., \& Strick, P.L. (2001). Imaging the premotor areas. Curr Opin Neurobiol, 11(6), 663-672.

Portney, L.G., \& Watkins, M.P. (2000). Foundations of clinical research: Applications to practice. Prentice Hall, Inc.

Rickards, T., Taub, E., Sterling, C., Graham, M.J., Barghi, A., Uswatte, G., \& Mark, V.W. (2012). Brain parenchymal fraction predicts motor improvement following intensive task-oriented motor rehabilitation for chronic stroke. Restor Neurol Neurosci, 30(5), 355-361.

Sergi, F., Drebs, H.I., Grossier, B., Rykman, A., Guglielmelli, E., Volpe, B.T., \& Schaechter, J.D. (2011). Predicting efficacy of robot-aided rehabilitation in chronic stroke patients using an MRI-compatible robotic device. Conf Proc IEEE Eng Med Biol Soc, 2011, 7470-7473.
Sitaram R., Viet R., Stevens B., Caria A., Gerloff C., Birbaumer N., Hummel F. (2012). Acquired control of ventral premotor cortex activity by feedback training. Neural Rehabilitation and Neural Repair 26(3), 256-265

Small, S.L., Hlustik, P., Noll, D.C., Genovese, C., \& Solodkin, A. (2002). Cerebellar hemispheric activation ipsilateral to the paretic hand correlates with functional recovery after stroke. Brain, 125(Pt 7), 1544-1557.

Strutton, P.H., Beith, I.D., Theodorou, S., Catley, M., McGregor, A.H., \& Davey, N.J. (2004). Corticospinal activation of internal oblique muscles has a strong ipsilateral component and can be lateralised in man. Exp Brain Res, 158(4), 474-479.

von, L.F., Hofer, S., Kaus, J., Merboldt, K.D., Rothkegel, H., Schweizer, R., Liebetanz, D., Frahm, J., \& Paulus, W. (2009). Efficacy of EMG-triggered electrical arm stimulation in chronic hemiparetic stroke patients. Restor Neurol Neurosci, 27(3), 189-197.

Ward, N.S., Brown, M.M., Thompson, A.J., \& Frackowiak, R.S. (2003). Neural correlates of outcome after stroke: A cross-sectional fMRI study. Brain, 126(Pt 6), 1430-1448

Wassermann, E.M., Pascual-Leone, A., \& Hallett, M. (1994). Cortical motor representation of the ipsilateral hand and arm. Exp Brain Res, 100(1), 121-132.

Yao, J., Chen, A., Carmona, C., \& Dewald, J.P. (2009). Cortical overlap of joint representations contributes to the loss of independent joint control following stroke. Neuroimage, 45(2), 490-499.

Zemke, A.C., Heagerty, P.J., Lee, C., \& Cramer, S.C. (2003). Motor cortex organization after stroke is related to side of stroke and level of recovery. Stroke, 34(5), e23-e28. 